\title{
Disruptions of the Human Connectome Associated With Hemispatial Neglect
}

Sadhvi Saxena, MS, MHS,* Zafer Keser, MD,* Chris Rorden, PhD, Leonardo Bonilha, MD, PhD,
Julius Fridriksson, PhD, Alexandra Walker, MA, and Argye Elizabeth Hillis, MD, MA

Neurology ${ }^{\circledR}$ 2022;98:e107-e114. doi:10.1212/WNL.0000000000013050

\section{Abstract}

\section{Background and Objectives}

Hemispatial neglect is a heterogeneous and complex disorder that can be classified by frame of reference for "left" vs "right," including viewer-centered neglect (VCN, affecting the contralesional side of the view), stimulus-centered neglect (SCN, affecting the contralesional side of the stimulus, irrespective of its location with respect to the viewer), or both. We investigated the effect of acute stroke lesions on the connectivity of neural networks that underlie VCN or SCN.

\section{Methods}

A total of 174 patients within 48 hours of acute right hemispheric infarct underwent a detailed hemispatial neglect assessment that included oral reading, scene copy, line cancellation, gap detection, horizontal line bisection tests, and MRI. Each patient's connectivity map was generated. We performed a linear association analysis between network connectivity strength and continuous measures of neglect to identify lesion-induced disconnections associated with the presence or severity of VCN and SCN. Results were corrected for multiple comparisons.

\section{Results}

About $42 \%$ of the participants with right hemisphere stroke had at least one type of neglect. The presence of any type of neglect was associated with lesions to tracts connecting the right inferior parietal cortex, orbitofrontal cortex, and right thalamus to other right-hemispheric structures. VCN only was strongly associated with tracts connecting the right putamen to other brain regions and tracts connecting right frontal regions with other brain regions. The presence of both types of neglect was most strongly associated with tracts connecting the right inferior and superior parietal cortex to other brain regions and those connecting left or right mesial temporal cortex to other brain regions.

\section{Discussion}

Our study provides new evidence for the specific white matter tracts where disruption can cause hemispatial neglect in a relatively large number of participants and homogeneous time after onset. We obtained MRI and behavioral testing acutely, before the opportunity for rehabilitation or substantial recovery.

\section{Classification of Evidence}

This study provides Class II evidence that damage to specific white matter tracts identified on MRI are associated with the presence of neglect following right hemispheric stroke.

\author{
Correspondence \\ Dr. Hillis \\ argye@jhmi.edu
}

RELATED ARTICLE

Editorial

What's Left in Neglect

Research?

Page 53

MORE ONLINE

III Class of Evidence

Criteria for rating

therapeutic and diagnostic

studies

NPub.org/coe

\footnotetext{
*Sadhvi Saxena and Zafer Keser contributed equally to this work as co-first authors.

From the Departments of Neurology (S.S., Z.K., A.W., A.E.H.) and Physical and Medicine \& Rehabilitation (A.E.H.), Johns Hopkins University School of Medicine, Baltimore, MD; Departments of Psychology (C.R.) and Communication Sciences and Disorders (J.F.), University of South Carolina, Columbia; Department of Neurology (L.B.), Medical University of South Carolina, Charleston; and Department of Cognitive Science (A.E.H.), Johns Hopkins University, Baltimore, MD.

Go to Neurology.org/N for full disclosures. Funding information and disclosures deemed relevant by the authors, if any, are provided at the end of the article. 


\section{Glossary}

CLSM = connectome-based lesion-symptom mapping; DWI = diffusion-weighted imaging; MNI = Montreal Neurological Institute; ROI = region of interest; $\mathbf{S C N}=$ stimulus-centered neglect; T1W = T1-weighted; TE = echo time; TR = repetition time; VCN = viewer-centered neglect.

Hemispatial neglect is a heterogeneous, complex syndrome that manifests as distinct, spatially specific impairments. One classification is based on the reference frame in which attention is distributed. ${ }^{1}$ That is, some individuals have viewercentered neglect (VCN), with deficits on the contralesional side of space defined by the viewer, such as the visual field, head, or body. ${ }^{2}$ Others have stimulus-centered neglect (SCN), with deficits on the contralesional side of the stimulus, irrespective of the side of the viewer. ${ }^{3}$ These types of neglect affect daily functions like reading and dressing in different ways and require distinct approaches to rehabilitation. ${ }^{1-3}$

Several studies have demonstrated that VCN and SCN occur independently of one another and reflect damage to different brain areas. ${ }^{4}$ Specifically, more dorsal cortical regions (frontal, parietal, superior temporal) are critical for encoding visual stimuli with respect to the viewer, or $\mathrm{VCN} .^{5-8}$ In contrast, more ventral cortical regions and deeper, subcortical areas are important for encoding stimuli irrespective of their location, causing SCN. ${ }^{4,7-11}$ White matter integrity is associated with the presence and severity of each type of deficit, ${ }^{12}$ suggesting that $\mathrm{VCN}$ and $\mathrm{SCN}$ result from distinct, anatomical disruptions in brain regions that are integrated in a distributed network. However, the specific white matter tracts that comprise the dorsal and ventral networks that, when damaged, can cause VCN or SCN have not yet been identified.

The human connectome allows one to visualize the sum of all structural and functional connections between neurons throughout the brain. ${ }^{13}$ We used a recently described and demonstrably effective lesion mapping approach ${ }^{14,15}$ to examine the lesion-induced effect on the structural connectivity of neural networks that underlie VCN or SCN. We studied patients with behavioral testing and imaging acutely after stroke to identify deficits before the opportunity for reorganization or recovery. We hypothesized that acute VCN is associated with damage to the network connecting regions of the dorsal visual stream and that acute SCN is associated with damage to the network connecting regions of the ventral visual stream.

The primary research question we addressed was as follows: Damage to what specific white matter tracts identified on MRI are associated with the presence of VCN and SCN following right hemispheric stroke?

\section{Methods}

\section{Participants}

We retrospectively analyzed prospectively collected data from 174 individuals (a convenience sample) who were admitted to the Johns Hopkins Hospital or Johns Hopkins Bayview Medical Center with an acute, ischemic right hemisphere stroke and tested within 48 hours of stroke onset. Patients were studied acutely after stroke to identify deficits before substantial recovery and to identify associated lesions before reorganization of structure-function relationships.

Participants were excluded from the analysis if they (1) had a history of other neurologic conditions affecting the brain, such as dementia; (2) had an impaired level of consciousness or ongoing sedation; (3) had uncorrected visual or hearing impairment; (4) had a psychiatric condition other than an affective disorder; (5) did not complete at least one test in a battery of neglect tests; (6) did not complete a diffusionweighted imaging (DWI) MRI; or (7) had bilateral stroke or a stroke of the cerebellum, medulla, or pons.

\section{Standard Protocol Approvals, Registrations, and Patient Consent Procedures}

This study was approved by the Johns Hopkins Medicine Institutional Review Board. Written informed consent was obtained from all participants.

\section{Assessment of Neglect}

Individuals included in this analysis completed at least one of the neglect tests described below. We attempted to administer the entire battery to all participants, but some were unable to complete all of the tests due to time constraints in the acute period (due to clinical assessments, rehabilitation services, and so on). These assessment materials are available at scorelab.jhmi.edu

1. Oral reading: reading aloud 30 words that were displayed over 2 columns, along with 5 sentences written across the page. All of these words can be made into another word by changing or omitting the first or last letters (e.g., darn could be read as "barn" or "dark" by erring on the first or last letter; rant could be read as "ant" or "ran" by omitting the first or last letter). Words were scored as neglect errors if the response preserved 1 or more letters at the end (right side) of the word and were incorrect on 1 or more letters at beginning (left side) of the word (e.g., rant read as "ant" or "pant" or even "hint" or "bit."

2. Scene copy: copying of the "Ogden scene," consisting of a tree, a fence, a house, and a second tree presented from left to right. ${ }^{16}$ The picture had 36 total components (pen strokes) in total, with 16 components on the left side and 20 on the right side of the page. Within the house and the 2 trees, 14 components were on the left and 14 components on the right side of the stimuli. Each omitted component 
was scored as an error; misplaced or distorted components were scored as half an error.

3. Line cancellation ${ }^{17}$ : crossing out all of the 28 vertical lines presented at the midsagittal plane and at $45^{\circ}$ both right and left of the viewer's midsagittal plane.

4. Gap detection ${ }^{18}$ : circling all complete circles and crossing out (with an $\mathrm{X}$ ) all of the circles with gaps. There were 30 circles total, with 15 circles on each side of the page. Ten circles had a gap on the left side and 10 had a gap on the right side. The remaining stimuli were full circles. Errors included stimuli either omitted (VCN) or marked incorrectly (SCN).

5. Horizontal line bisection: placing a mark at the center of a horizontal line that was presented at the midsagittal plane and at $45^{\circ}$ both right (ipsilesional position) and left (contralesional position) of the viewer's midsagittal plane. The extent of error in each viewpoint was determined by percent deviation to the right side from the center of the page.

We dichotomously defined VCN, SCN, and both types of neglect. We defined VCN as 2 or more errors on the contralesional (left) side than the ipsilesional (right) side of the page on the gap detection tests (failure to mark the stimuli at all), 2 or more on the left than the right side of the page in the copy scene test, 2 or more errors on the left position than the right position with respect to the viewer on the line cancellation test, or 2 or more errors in reading words on the left than the right column in the oral reading test. For the line bisection test, we defined VCN as greater than $10 \%$ deviation on the contralesional position than the ipsilesional position.

We defined SCN as 2 or more errors on the contralesional (left) side than the ipsilesional (right) side of stimuli in the gap detection test (failure to detect gaps in circles with gaps on left or right), the copy scene task (omitting components on the contralesional side of the house and trees), or oral reading (errors on the left vs the right sides of words). For the line bisection test, we defined SCN as greater than $10 \%$ deviation in all 3 positions, irrespective of the viewpoint (i.e., without a greater than $10 \%$ difference on the contralesional position compared to the ipsilesional position).

To determine VCN severity, we calculated the average number of errors (failure to mark the stimuli) on the contralesional side of the page in the gap detection and scene copy tests. We determined SCN severity by calculating the average number of failures to detect the contralesional (left) gap on circles, irrespective of the side of the page, in these tests. We used only the gap detection and scene copy tests because they were most frequently completed by participants (172/174 and 163/174, respectively). Due to non-normal skewed distribution of the VCN and SCN errors in gap detection, we dichotomized errors into mild ( $0-2$ errors) and severe ( 3 or more errors) groups for neuroimaging analysis. Examples of responses showing VCN and SCN are shown in Figure 1.

\section{Imaging}

\section{Image Acquisition and Preprocessing}

All participants underwent MRI in the acute hospitalization period, in accordance with acute stroke standard of care at Johns Hopkins. The images obtained clinically were used in this study for lesion quantification. Specifically, the imaging sequences used in this study were part of the clinical scan and included a 3D T1-weighted (T1W) sequence and a diffusionweighted imaging (DWI) sequence. MRI data were acquired on a 3T Siemens Trio scanner. 3D T1W images were acquired sagittally with slice thickness $1.5 \mathrm{~mm}$ and no gaps, repetition time (TR)/echo time (TE)/inversion time 2,300/35/900 ms, flip angle $9^{\circ}$, and field of view $256 \times 256$. DWI data were acquired axially with slice thickness $4 \mathrm{~mm}$ with no gaps, 3 directions, TR/TE 5,000/78 ms, flip angle $90^{\circ}$, field of view $256 \times 256$ and $\mathrm{b}=1,000 \mathrm{~s} / \mathrm{mm}^{2}$. The diffusion MRI was used for lesion quantification. Technicians trained in lesion drawing and in neuroanatomy manually delineated the area of restricted diffusion in the B0 image, with confirmation through the apparent diffusion coefficient image. All lesion tracings were checked and confirmed by a stroke neurologist (A.E.H.).

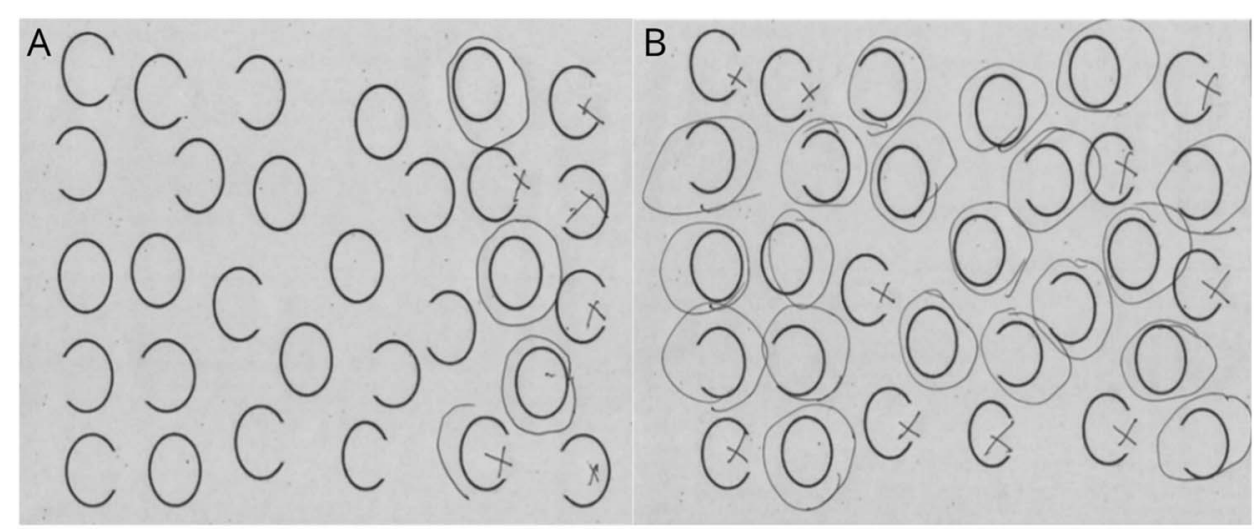

(A) Left viewer-centered neglect (VCN) (omission of stimuli in left view). (B) Left stimulus-centered neglect (SCN) (failure to detect gaps on left side of each stimulus, on both sides of the view). 
Table 1 Demographics

\begin{tabular}{llll}
\hline Group & Age, $\mathbf{y}$, mean (SD) & \% Female & \% Left-handed or ambidextrous $^{\mathbf{a}}$ \\
\hline Any neglect $(\mathbf{n}=\mathbf{7 3})$ & $64.1(14.2)$ & 52 & 9 \\
\hline No neglect $(\mathbf{n}=\mathbf{1 0 1})$ & $57.4(13.3)$ & 49 & 5 \\
\hline Group difference by 2-tailed $\boldsymbol{t}$ test or $\boldsymbol{\chi}^{\mathbf{2}}$ & $\begin{array}{l}t=3.2 \\
p=0.002\end{array}$ & $\begin{array}{l}\chi^{2}=0.006 \\
p=0.93\end{array}$ & $\begin{array}{l}\chi^{2}=0.21 \\
p=0.65\end{array}$ \\
\hline
\end{tabular}

${ }^{a}$ Handedness not reported by 8 participants with any neglect and 5 participants with no neglect.

Once the lesion tracing was obtained (the lesion "mask"), we employed an enantiomorphic normalization approach to normalize the lesion mask into standard space. This step was performed using the software NiiStat (github.com/neurolabusc/NiiStat), which leverages routines from the software package SPM software (fil.ion.ucl.ac.uk/spm/). NiiStat is an in-house MATLAB program, which includes routines for enantiomorphic normalization in accordance with Nachev et al. ${ }^{19}$ In brief, the enantiomorphic normalization enables accurate normalization to standard Montreal Neurological Institute (MNI) space by filling the parts of brain with lesions with data from the spared contralateral hemisphere. Once the images are normalized to standard space, the normalization matrix is then applied to the lesion mask, yielding a lesion mask in standard MNI space.

\section{Effect of Lesions on Connectivity}

To assess the effect of lesions on connectivity, we employed an indirect measure of the lesion's effect on structural connectivity by evaluating the white matter fibers that would have been damaged by the individual lesion. This approach utilizes an atlas of white matter connectivity obtained from diffusion tensor imaging tractography from multiple individuals (a connectogram), thereby providing a representative view of typical white matter pathways in the normal population. The lesion image is then overlaid into this 3D map of connectivity and the fibers whose paths intersect the lesion are considered to be damaged. That is, the atlas of white matter connectivity was derived from DTI of healthy controls, and then we used DWI lesions overlaid on this atlas to determine the tracts that were lesioned.
The main advantage of this approach is to permit a more detailed and thorough evaluation of the effect of the lesion not only with regards to the area of necrosis or gliosis, but also relative to the remote disconnection caused by the lesion based on the white matter damage and the fibers that are destroyed. This more modern and thorough approach in lesion quantification is based on the well-known phenomenon in neurology called diaschisis, where areas typically spared by the lesion can be disconnected and therefore dysfunctional. ${ }^{20}$ In the context of other cognitive deficits from stroke-for example, aphasia-this is increasingly recognized as an important determinant of clinical symptoms. ${ }^{21-23}$

The main disadvantage of this method is that it constitutes an indirect measurement of white matter connectivity. Preferably, the individual's own spared connectivity would have been performed using high-resolution diffusion tensor imaging tractography as stroke survivors may not have the same preservation of white matter fibers compared with healthy controls. Nonetheless, this is a reasonable compromise because (1) high-resolution diffusion tensor imaging is seldom available or feasible in the acute stroke period; and (2) the main effects being investigated are related to large pathways, which are consistent across individuals even if individual variability related to cardiovascular risks factors is present.

Specifically, this approach was accomplished as follows: (1) we employed a tractogram from the Human Connectome Project including data from 842 individuals in standard space (Ref); (2) the fibers included in the tractogram (.fib format) file were read into MATLAB using a modified version of trk

Table 2 Lesion Load and Vascular Risk Factors by Group

\begin{tabular}{llllll}
\hline Group & Lesion load & DM & HTN & Smoking at time of stroke & Afib \\
\hline Any neglect $(\mathbf{n}=\mathbf{7 3})$ & $3.8(5.1)$ & 56 & 86 & 30 & 32 \\
\hline No neglect $(\mathbf{n}=\mathbf{1 0 1})$ & $1.5(3.4)$ & 50 & 81 & 32 & 24 \\
\hline Group difference by 2-tailed $\boldsymbol{t}$ test or $\boldsymbol{\chi}^{\mathbf{2}}$ & $\begin{array}{l}t=3.6 \\
p=0.0004\end{array}$ & $\begin{array}{l}\chi^{2}=0.44 \\
p=0.50\end{array}$ & $\begin{array}{l}\chi^{2}=0.61 \\
p=0.43\end{array}$ & $\begin{array}{l}\chi^{2}=0.08 \\
p=0.78\end{array}$ & $\begin{array}{l}\chi^{2}=1.2 \\
p=0.27\end{array}$ \\
\hline
\end{tabular}

Abbreviations: Afib = atrial fibrillation; DM = diabetes mellitus; HTN = hypertension.

Values are mean (SD) or \%.

${ }^{a}$ Lesion load $=$ lesion volume/intracranial volume $\times 100$ (\% of intracranial volume) 
read (github.com/johncolby); (3) the Desikan atlas (eTable 1, links.lww.com/WNL/B655) (Ref) including gray matter regions of interest (ROIs) was resliced into the same space as the tractogram; (4) the normalized lesion masks were also resliced into the same space; (5) the fibers connecting each possible pair of ROIs in the Desikan atlas were computed, generating a $2 \mathrm{D}$ matrix (the normal connectome) (fibers that passed or ended in the ROI voxels were included as connecting that ROI); (6) the fibers that passed through the lesion were considered lesioned; (7) for each individual, the normal connectome minus the fibers (and their pairwise connections) was constructed, thus generating a $2 \mathrm{D}$ connectivity matrix per individual representing their spared connectivity. Likewise, for each subject, a map of disconnection was generated (the normal connectogram - the individual spared connectogram). These steps were performed using in-house routines written in MATLAB.

An example of tracts coregistered in the same space as Desikan ROIs can be seen in eFigure 1 (links.lww.com/WNL/ B655).

\section{Relationship Between Loss Connectivity and Neglect}

Once each participant's connectivity map was calculated, we performed connectivity-based analyses to evaluate the presence of connections with neglect symptoms. This approach is similar to previously described methods of connectomelesion symptom mapping (connectome-based lesionsymptom mapping [CLSM]), ${ }^{24}$ where a linear model is used to evaluate the linear association between strength of connectivity (presence and number of fibers in each ROI-ROI connection) with continuous measures of neglect. The main difference here was that each participant's ROI pairwise disconnection values (i.e., the normal connectogram - the individual's spared connectivity) was used. Only connections lesioned in at least $25 \%$ of the individuals were used in these statistical analyses and the critical $p$ value (corrected for the number of multiple statistical comparisons) was set as the number of $0.05 /$ connections being tested.

\section{Data Availability}

The authors documented all data, methods, and materials used to conduct the research presented. Anonymized data pertaining to the research presented will be made available by request from qualified investigators.

\section{Results}

\section{Participant Characteristics}

Demographic characteristics of patients with and without neglect are shown in Table 1. Lesion load and vascular risk factors are shown in Table 2. Of the 174 participants, 73 were found to have any type of neglect. As shown in the tables, participants with neglect were significantly older and had greater lesion load (lesion volume as a percentage of intracranial volume; Table 2). However, there were no significant differences between those with and without neglect in percentage female or left-handed or ambidextrous (Table 1), or in percentage with vascular risk factors of diabetes, hypertension, smoking at the time of stroke, or atrial fibrillation (Table 2).

\section{Behavioral Results}

Among the 174 participants, 26 (15\%) had VCN only, 15 (9\%) had SCN only, and 32 (18\%) had both types of neglect. Thus, $73(42 \%)$ of the participants with right hemisphere stroke had one or more types of neglect (any neglect).

\section{Neuroimaging Results}

Overall, participants had 1,604 connections, with lesions in at least $25 \%$ of the participants. The number of connections lesioned across all participants is shown in Figure 2.

When comparing individuals with neglect vs those without neglect, using an edgewise $t$ test, that is, which pathways had

Figure 2 Locations of Lesion-Induced Disconnections (Disconnectogram)
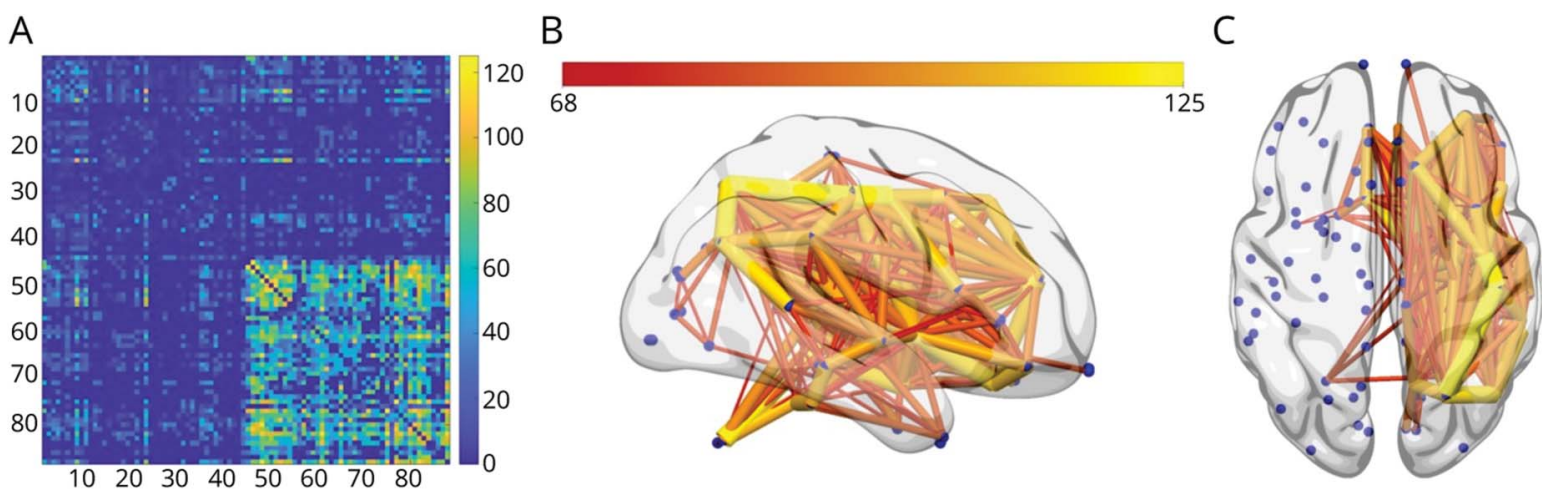

(A) The 2D connectivity matrix demonstrates the locations of disconnections induced by lesions (statistically associated with lesions in that location) across all participants on a color-coded scale (scale 0-125+ participants). The rows and columns are numbered in accordance with the Desikan Atlas (eTable 1 , links. lww.com/WNL/B655). (B, C) The locations of lesion-induced disconnections in the entire cohort are also presented in the 3D brain template images. The edges are colored in accordance with how many participants had lesions involving that pathway (color-coded scale 68-125+ participants). 
fewer connections in individuals with neglect, we observed that neglect was associated with lesions to certain connections (Figure 3; eTable 1, eTable 2, and eTable 3, links.lww.com/ $\mathrm{WNL} / \mathrm{B} 655)$. We report only associations that remained significant after multiple corrections with Bonferroni correction $(p<0.000031)$. In order to investigate the connectivity profiles with increased severity of neglect in VCN and SCN, we dichotomized errors in gap detection into mild (0-2 errors) and severe ( 3 or more errors). Rather than classical correlation analysis, we utilized dichotomized analysis for the non-normal skewed distribution of gap detection errors (see eFigure 1 and eFigure 2) in our cohort. Edgewise $t$ test was utilized to determine connectivity profiles in mild and severe neglect groups. There was no difference in connectivity profiles between mild and severely affected SC groups. eTable 4

Figure 3 Disconnections Associated With Neglect

\section{A. Any form of neglect vs no neglect}
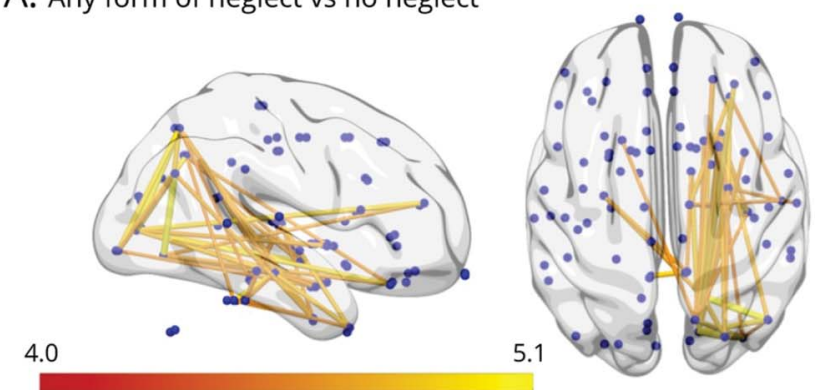

B. VCN vs other forms of neglect or no neglect

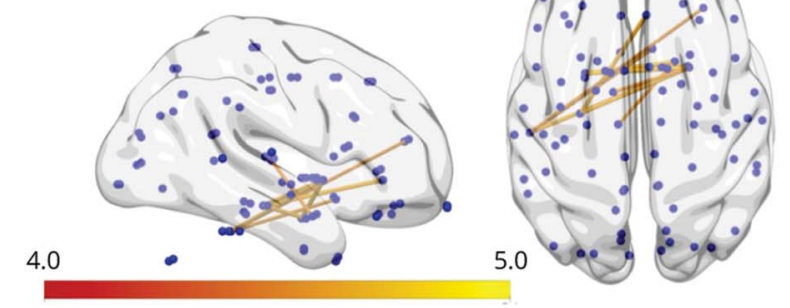

C. VCN combined with SCN vs no neglect

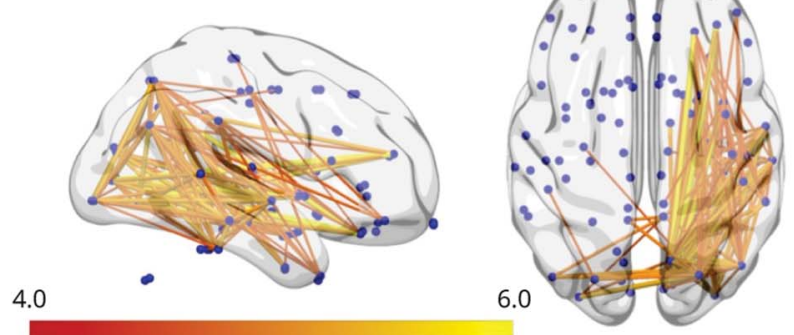

These disconnectogram atlases each provide a summary of lesion-induced disconnections that were associated with the presence of a given type of neglect (determined by using an edgewise $t$ test). They are illustrated in 3D brain template images with color-coded scales of $t$ value (between 4 and 5.1). (A) Disconnectogram atlas associated with any type of neglect (viewercentered neglect [VCN] or stimulus-centered neglect [SCN]). (B) Disconnectogram atlas associated with VCN only. (C) Disconnectogram atlas associated with the presence of both types of neglect (SCN and VCN combined). summarizes the significantly fewer connections in more severe VCN compared to mild VCN.

\section{Classification of Evidence}

This study provides Class II evidence that damage to specific white matter tracts identified on MRI are associated with the presence of neglect following right hemispheric stroke.

\section{Discussion}

The role of disrupted white matter tracts in causing the clinical syndrome of neglect has been known for some time.25,26 We used a new approach to identify specific structural connections that when damaged can cause specifically VCN, as well as any type of neglect, or both VCN and SCN.

The presence of any type of neglect (Table 1, Figure 3A) was, as expected from previous literature, associated with lesions to tracts connecting right inferior parietal cortex, ${ }^{9,27-29}$ orbitofrontal cortex, ${ }^{9,30}$ and right thalamus ${ }^{31-33}$ to other brain regions primarily in the right hemisphere. Likewise, the role of tracts connecting right inferior temporal, occipital, and fusiform cortex might be expected from studies of lesions associated with SCN. ${ }^{4,7,9-11}$ Less expected, however, was the importance of white matter tracts connecting left or right mesial temporal cortex, including hippocampus, amygdala, entorhinal cortex, and parahippocampal cortex, to other brain regions. These areas are often implicated in disorders of new learning and recall. Corbetta and Schulman ${ }^{25}$ have proposed that the clinical syndrome of neglect requires 2 deficits: one of sustained attention and one of spatially specific processing. It is also possible that a deficit in learning/recall can contribute to the manifestations of neglect, particularly as our clinical neglect tasks required retention of the instructions. That is, it is plausible that patients with impaired retention started to respond to stimuli on the ipsilesional side (due to a spatially specific bias), but then forgot the instructions, leading to a failure to respond to additional stimuli in line cancellation, copying, or gap detection.

Viewer-centered neglect only (Table 2, Figure 3B) was strongly associated with tracts connecting the right putamen to other brain regions, as well as tracts connecting right frontal regions with other brain regions. A critical role of right putamen lesions in causing neglect has been previously demonstrated by Karnath et al. ${ }^{8}$ Likewise, lesions in right frontal areas have been implicated in left VCN., ${ }^{9,34}$

The presence of both types of neglect (eTable 3, links.lww. com/WNL/B655; Figure 3C) was most strongly associated with tracts connecting right inferior and superior parietal cortex to other brain regions, as well as those connecting left or right mesial temporal cortex to other brain regions, consistent with a critical role of right temporoparietal cortex in neglect, ${ }^{29-32}$ as well as a potential role of learning and recall mechanisms. A few tracts connecting right inferior temporal/ 
occipital regions to other brain regions, consistent with the role of these areas in $\mathrm{SCN}^{4,7,9-11}$ were also identified in association with both types of neglect.

Finally, the severity of VCN (eTable 4 and eTable 5, links. lww.com/WNL/B655) was strongly associated with tracts associated with any or both types of neglect, including those connecting areas frequently associated with neglect in parietal cortex and other brain regions and tracts connecting mesial temporal lobe structures with other brain regions.

We did not identify any tracts where lesions were associated with presence or severity of left SCN alone, likely because of inadequate power. Other studies have revealed distinct lesions or areas of hypoperfusion associated with these different forms of neglect. ${ }^{9,10,35}$ Left SCN in isolation is relatively uncommon after right hemisphere stroke, although right $\mathrm{SCN}$ is more common than right VCN after left hemisphere stroke. ${ }^{36}$

The novel results reported here indicate the importance of lesions affecting white matter tracts connecting not only areas known to cause neglect (e.g., parietal, frontal, and temporal cortex) and thalamus, but also right putamen and bilateral mesial temporal structures with other brain regions, in the clinical syndrome of hemispatial neglect. Lesions involving tracts connecting right putamen with other structures were particularly important in causing isolated right VCN.

Strengths of the study include the relatively large number of participants (174), the relatively homogeneous time post onset at which MRI and behavioral testing were obtained, and the novel approach to identifying white matter tracts where disruption can cause hemispatial neglect. Limitations of this study include the small number of participants with SCN alone (which may have undermined our ability to identify disconnections that lead to this form of neglect), as 15 participants with SCN may not have provided adequate power to identify significant associations. Other limitations include the inability to complete all 4 neglect tests in all patients and lack of diffusion tensor imaging that would allow direct evaluation of disruptions of white matter tracts in our patients. Furthermore, the neglect tests we used, while they have strong face validity and are widely used to assess for neglect, have limited established psychometric properties. Some errors could have been due to attentional factors that were not due to the lesion itself, although we excluded patients with reduced level of consciousness.

Despite its limitations, this study provides support for the general proposal that a network of brain regions, including inter- and intrahemispheric white matter connections, is critical for performance on spatial attention tasks. Lesions that disrupt one or more of these connections can result in VCN or a combination of $\mathrm{VCN}$ and $\mathrm{SCN}$, and greater damage to the connections can cause more severe $\mathrm{VCN}$, in acute right hemisphere stroke, before recovery through reorganization of structure-function relationships.

\section{Acknowledgment}

The authors thank the study participants and the NIH for support. The content is solely the responsibility of the authors and does not necessarily represent the views of the NIH.

\section{Study Funding}

This work was supported by the NIH (National Institute of Neurologic Disorders and Stroke and National Institute on Deafness and Communication Disorders) through award R01 DC05466 (PI: Hillis) and P50 014664 (PI: Fridriksson).

\section{Disclosure}

The authors report no disclosures relevant to the manuscript. Go to Neurology.org/N for full disclosures.

\section{Publication History}

Received by Neurology April 19, 2021. Accepted in final form November 2, 2021.

Appendix Authors

\begin{tabular}{lll}
\hline Name & Location & Contribution \\
\hline $\begin{array}{ll}\text { Sadhvi } \\
\text { Saxena, MS, }\end{array}$ & $\begin{array}{l}\text { Department of Neurology, } \\
\text { Johns Hopkins University } \\
\text { School of Medicine }\end{array}$ & $\begin{array}{l}\text { Drafting/revision of the } \\
\text { manuscript for content, } \\
\text { including medical writing for } \\
\text { content; major role in the } \\
\text { acquisition of data; study } \\
\text { concept or design; analysis } \\
\text { or interpretation of data }\end{array}$ \\
\end{tabular}

\begin{tabular}{lll}
\hline $\begin{array}{l}\text { Zafer Keser, } \\
\text { MD }\end{array}$ & $\begin{array}{l}\text { Department of Neurology, } \\
\text { Johns Hopkins University } \\
\text { School of Medicine }\end{array}$ & $\begin{array}{l}\text { Drafting/revision of the } \\
\text { manuscript for content, } \\
\text { including medical writing for } \\
\text { content; study concept or } \\
\text { design; analysis or } \\
\text { interpretation of data }\end{array}$ \\
\end{tabular}

Chris Department of Psychology, Study concept or design;

Rorden, PhD University of South Carolina analysis or interpretation of data

\begin{tabular}{|c|c|c|}
\hline $\begin{array}{l}\text { Leonardo } \\
\text { Bonilha, } \\
\text { MD, PhD }\end{array}$ & $\begin{array}{l}\text { Department of Neurology, } \\
\text { Medical University of South } \\
\text { Carolina }\end{array}$ & $\begin{array}{l}\text { Study concept or design; } \\
\text { analysis or interpretation of } \\
\text { data }\end{array}$ \\
\hline $\begin{array}{l}\text { Julius } \\
\text { Fridriksson, } \\
\text { PhD }\end{array}$ & $\begin{array}{l}\text { Department of } \\
\text { Communication Sciences } \\
\text { and Disorders, University of } \\
\text { South Carolina }\end{array}$ & $\begin{array}{l}\text { Study concept or design; } \\
\text { analysis or interpretation of } \\
\text { data }\end{array}$ \\
\hline $\begin{array}{l}\text { Alexandra } \\
\text { Walker, MA }\end{array}$ & $\begin{array}{l}\text { Department of Neurology, } \\
\text { Johns Hopkins University } \\
\text { School of Medicine }\end{array}$ & $\begin{array}{l}\text { Drafting/revision of the } \\
\text { manuscript for content, } \\
\text { including medical writing for } \\
\text { content; major role in the } \\
\text { acquisition of data; analysis } \\
\text { or interpretation of data; } \\
\text { obtained additional data } \\
\text { requested by one of the } \\
\text { reviewers }\end{array}$ \\
\hline $\begin{array}{l}\text { Argye } \\
\text { Elizabeth } \\
\text { Hillis, MD, } \\
\text { MA }\end{array}$ & $\begin{array}{l}\text { Departments of Neurology } \\
\text { and Physical and Medicine \& } \\
\text { Rehabilitation, Johns } \\
\text { Hopkins University School of } \\
\text { Medicine; Department of } \\
\text { Cognitive Science, Johns } \\
\text { Hopkins University }\end{array}$ & $\begin{array}{l}\text { Drafting/revision of the } \\
\text { manuscript for content, } \\
\text { including medical writing for } \\
\text { content; major role in the } \\
\text { acquisition of data; study } \\
\text { concept or design; analysis } \\
\text { or interpretation of data }\end{array}$ \\
\hline
\end{tabular}




\section{References}

1. Caramazza A, Hillis AE. Spatial representation of words in the brain implied by studies of a unilateral neglect patient. Nature. 1990;346(6281):267-269.

2. Behrmann M, Geng JJ. What is 'left' when all is said and done? spatial coding and hemispatial neglect. In: The Cognitive and Neural Bases of Spatial Neglect. Oxford University Press; 2002:85-100.

3. Vallar G, Bottini G, Paulesu E. Neglect syndromes: the role of the parietal cortex. $A d v$ Neurol. 2003;93:293-319.

4. Hillis AE, Newhart M, Heidler J, Barker PB, Herskovits EH, Degaonkar M. Anatomy of spatial attention: insights from perfusion imaging and hemispatial neglect in acute stroke. J Neurosci. 2005;25(12):3161-3167.

5. Kenzie JM, Girgulis KA, Semrau JA, Findlater SE, Desai JA, Dukelow SP. Lesion sites associated with allocentric and egocentric visuospatial neglect in acute stroke. Brain Connectivity. 2015;5(7):413-422.

6. Chen P, Caulfield MD, Hartman AJ, O’Rourke J, Toglia J. Assessing viewer-centered and stimulus-centered spatial bias: the $3 \mathrm{~s}$ spreadsheet test version 1. Appl Neuropsychol Adult. 2017;24(6):532-539.

7. Suarez A, Saxena S, Oishi K, et al. Influence of age, lesion volume, and damage to dorsal versus ventral streams to viewer- and stimulus-centered hemispatial neglect in acute right hemisphere stroke. Cortex. 2020;126:73-82.

8. Karnath HO, Himmelbach M, Rorden C. The subcortical anatomy of human spatial neglect: putamen, caudate nucleus and pulvinar. Brain. 2002;125(pt 2):350-360.

9. Medina J, Kannan V, Pawlak MA, et al. Neural substrates of visuospatial processing in distinct reference frames: evidence from unilateral spatial neglect. J Cogn Neurosci. 2009;21(11):2073-2084

10. Shirani P, Thorn J, Davis C, et al. Severity of hypoperfusion in distinct brain regions predicts severity of hemispatial neglect in different reference frames. Stroke. 2009; 40(11):3563-3566.

11. Verdon V, Schwartz S, Lovblad KO, Hauert CA, Vuilleumier P. Neuroanatomy of hemispatial neglect and its functional components: a study using voxel-based lesionsymptom mapping. Brain. 2010;133(pt 3):880-894.

12. Vaessen MJ, Saj A, Lovblad KO, Gschwind M, Vuilleumier P. Structural white-matter connections mediating distinct behavioral components of spatial neglect in right brain-damaged patients. Cortex. 2016;77:54-68

13. Sporns O, Tononi G, Kötter R. The human connectome: a structural description of the human brain. PLOS Comput Biol. 2005;1(4):e42.

14. Boes AD, Prasad S, Liu H, et al. Network localization of neurological symptoms from focal brain lesions. Brain. 2015;138(pt 10):3061-3075.

15. Fox MD. Mapping symptoms to brain networks with the human connectome. N Engl J Med. 2018;379(23):2237-2245.

16. Ogden JA. Contralesional neglect of constructed visual images in right and left braindamaged patients. Neuropsychologia. 1985;23(2):273-277.

17. Albert ML. A simple test of visual neglect. Neurology. 1973;23(6):658-664.
18. Ota H, Fujii T, Suzuki K, Fukatsu R, Yamadori A. Dissociation of body-centered and stimulus-centered representations in unilateral neglect. Neurology. 2001;57(11): 2064-2069.

19. Nachev P, Coulthard E, Jäger HR, Kennard C, Husain M. Enantiomorphic normalization of focally lesioned brains. Neuroimage. 2008;39(3):1215-1226.

20. Feeney DM, Baron JC. Diaschisis. Stroke. 1986;17(5):817-830.

21. Fridriksson J, den Ouden DB, Hillis AE, et al. Anatomy of aphasia revisited. Brain. 2018;141(3):848-862.

22. Yourganov G, Fridriksson J, Rorden C, Gleichgerrcht E, Bonilha L. Multivariate connectome-based symptom mapping in post-stroke patients: networks supporting language and speech. J Neurosci. 2016;36(25):6668-6679.

23. Bonilha L, Fridriksson J. Subcortical damage and white matter disconnection associated with non-fluent speech. Brain. 2009;132(pt 6):e108.

24. Gleichgerrcht E, Fridriksson J, Rorden C, Bonilha L. Connectome-based lesionsymptom mapping (CLSM): a novel approach to map neurological function. Neuroimage Clin. 2017;16:461-467.

25. Corbetta M, Shulman GL. Spatial neglect and attention networks. Annu Rev Neurosci. 2011;34:569-599.

26. Bartolomeo P, Thiebaut de Schotten M, Doricchi F. Left unilateral neglect as a disconnection syndrome. Cereb Cortex. 2007;17(11):2479-2490.

27. Heilman KM, Van Den Abell T. Right hemisphere dominance for attention: the mechanism underlying hemispheric asymmetries of inattention (neglect). Neurology. 1980;30(3):327-330.

28. Mort DJ, Malhotra P, Mannan SK, et al. The anatomy of visual neglect. Brain. 2003; 126(pt 9):1986-1997.

29. Mesulam MM. Spatial attention and neglect: parietal, frontal and cingulate contributions to the mental representation and attentional targeting of salient extrapersonal events. Philos Trans R Soc Lond B Biol Sci 1999;354(1387):1325-1346.

30. Heilman KM, Valenstein E. Frontal lobe neglect in man. Neurology. 1998;50(5) 1202 -and 5 pages following.

31. Watson RT, Valenstein E, Heilman KM. Thalamic neglect: possible role of the medial thalamus and nucleus reticularis in behavior. Arch Neurol. 1981;38(8):501-506.

32. Watson RT, Heilman KM. Thalamic neglect. Neurology. 1979;29(5):690-694.

33. Sebastian R, Schein MG, Davis C, et al. Aphasia or neglect after thalamic stroke: the various ways they may be related to cortical hypoperfusion. Front Neurol. 2014;5:231.

34. Hillis AE. Neurobiology of unilateral spatial neglect. Neuroscientist. 2006;12(2) 153-163.

35. Khurshid S, Trupe LA, Newhart M, et al. Reperfusion of specific cortical areas is associated with improvement in distinct forms of hemispatial neglect. Cortex. 2012; 48(5):530-539.

36. Kleinman JT, Newhart M, Davis C, Heidler-Gary J, Gottesman RF, Hillis AE. Right hemispatial neglect: frequency and characterization following acute left hemisphere stroke. Brain Cogn. 2007;64(1):50-59. 


\section{Neurology}

\section{Disruptions of the Human Connectome Associated With Hemispatial Neglect \\ Sadhvi Saxena, Zafer Keser, Chris Rorden, et al. \\ Neurology 2022;98; e107-e114 \\ DOI 10.1212/WNL.0000000000013050}

This information is current as of January 10, 2022

\section{Updated Information \& Services}

References

Subspecialty Collections

Permissions \& Licensing

Reprints including high resolution figures, can be found at: http://n.neurology.org/content/98/2/e107.full

This article cites 35 articles, 9 of which you can access for free at: http://n.neurology.org/content/98/2/e107.full\#ref-list-1

This article, along with others on similar topics, appears in the following collection(s):

All Cerebrovascular disease/Stroke

http://n.neurology.org/cgi/collection/all_cerebrovascular_disease_strok

Class II

http://n.neurology.org/cgi/collection/class_ii

Information about reproducing this article in parts (figures,tables) or in its entirety can be found online at:

http://www.neurology.org/about/about_the_journal\#permissions

Information about ordering reprints can be found online:

http://n.neurology.org/subscribers/advertise

Neurology ${ }^{\circledR}$ is the official journal of the American Academy of Neurology. Published continuously since 1951, it is now a weekly with 48 issues per year. Copyright (O) 2022 American Academy of Neurology. All rights reserved. Print ISSN: 0028-3878. Online ISSN: 1526-632X.

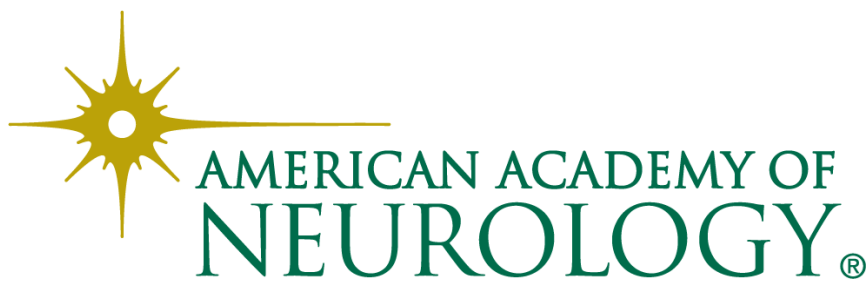

